\title{
Efficacy, Tolerability and Adverse Events of Single-Shot Intra-Articular Hyaluronic Acid Injection in Knee Osteoarthritis
}

\section{Shahid Hussain ${ }^{1 *}$, Hamid Rather ${ }^{1}$ and Asif Qayoom ${ }^{2}$}

${ }^{1}$ Department of Physical Medicine and Rehabilitation, Sheri-Kashmir Institute of Medical Sciences, Soura, Srinagar, Jammu \& Kashmir, India

2 Urgent Care Hospital, New Delhi, India

\section{Abstract}

Objective: The clinical experience with single intra-articular hyaluronic acid injection in knee OA is limited. The aim was to assess the therapeutic efficacy, tolerability and adverse events of single-shot intra-articular hyaluronic acid injection in knee OA.

Method: Between February 2008 and December 2010, forty eight (48) patients (Males=21, Females=27) with OA knees were enrolled in this prospective study. These patients had failed to respond adequately to conservative treatment including analgesics and rehabilitative modalities. The inclusion criteria were: 1) Resting visual analog scale pain of $>50 \mathrm{~mm}$ and 2) Radiographic evidence for osteoarthritis and at least one of the following three characteristics; Age $\geq 50$ yrs, Morning stiffness $\leq 30 \mathrm{~min}$ in duration and coarse crepitus on motion (as per American College of Rheumatology guidelines 1986). Functional scoring as per WOMAC and VAS for pain at rest and during walking was documented. Patient satisfaction was documented as per Linkert scale. Objective analysis included measurement of knee flexion-extension, circumference at the level of suprapatellar pouch and change in joint space width (Tibiofemoral joint). Patients received a single injection of Synvisc-One (consisting of $6 \mathrm{ml}$ hylan G-F 20). The patients were reviewed at 1 month, 3 months and 6 months post-injection with final analysis at 1 year. Use of only paracetamol, when required was allowed for concomitant analgesia but disallowed around the time of clinical evaluation.

Results: The mean age of the patients was $65 \pm 5$ years; mean duration of symptoms $5.4 \pm 1.5$ years and mean body mass index (BMI) $29.1 \mathrm{~kg} / \mathrm{m}^{2}$. Bilateral knees were involved in $69 \%$ of patients. Knee swelling and effusion was present in $70 \%$ and $31.5 \%$ patients, respectively. All the patients were available at final follow-up. The resting and walking VAS pain significantly improved from baseline after the injection (45 from 70 and 50 from 82 , respectively). There was significant improvement of pain and disability based on the WOMAC scores. Adverse events were recorded and included local pain and swelling, mild redness, and/or effusion in the knee. Based on "Knee Society Score" the overall efficacy was judged as excellent in $55 \%$, satisfactory in $43 \%$, and poor in $2 \%$. The beneficial effects stayed till 6 months but returned to baseline at last follow up at 1 year. Substantial improvement was noted in knee flexionextension and knee swelling $(p<0.05)$ but no change was observed in joint space width.

Conclusions: This study confirmed the therapeutic efficacy and safety of single-shot intra-articular injection of hyaluronic acid for the treatment of osteoarthritis of the knee. The pain relief and functional improvement lasts for up to 6 months. The procedure is tolerated well and associated with very few local adverse events. The need for concomitant analgesia is reduced.

Keywords: Hyaluronic Acid; Knee Osteoarthritis; Visco-supplimentation

\section{Introduction}

Osteoarthritis is the most common chronic joint disorder in the elderly mostly affecting the weight bearing joints of the lower limbs particularly the knees [1]. It is characterized by progressive deterioration and loss of articular cartilage and by reactive new bone formation at the joint margins [2]. Clinical manifestations include slowly developing joint pain, stiffness, and joint enlargement with limitations of motion. Knee osteoarthritis results from mechanical and idiopathic factors that alter the balance between degradation and synthesis of HA in synovial fluid and articular cartilage and subchondral bone.

There are several options for treating osteoarthritis of the knee including conservative (analgesics, physiotherapy/ physical modalities, weight-relieving braces, intra-articular injections) and surgical (osteotomy, arthroplasty) treatment.

First proposed by Balazs in 1993 visco-supplementation with intraarticular Hyaluronic acid (HA) injections has emerged as a viable treatment modality for nonoperative care of symptomatic osteoarthritis $[2,3]$.

Hyaluronic acid (HA) is a linear, long-chain, unbranched, hydrophilic, high molecular weight muco-polysaccharide forming a critical constituent component of the synovial fluid and of cartilage. It is an important contributor to maintain joint homeostasis [4]. In patients with osteoarthritis, synovial hyaluronic acid is depolymerized and cleared at higher rates than in normal individuals [2], leading to deterioration of joint cartilage and synovial fluid characteristics etc and manifesting clinically as pain and loss of function. Specially, there is a decrease in concentration and molecular weight of HA on OA $[5,6]$. Hence, intraarticular viscosupplementation with HA may restore normal biochemical properties/ characteristics in various joint structures, resulting in improved pain control and function [7].

*Corresponding author: Shahid Hussain, Department of Physical Medicine and Rehabilitation, Sheri-Kashmir Institute of Medical Sciences, Soura, Srinagar, Jammu \& Kashmir, India, Tel: 9419003100; E-mail: Drshahid2006@gmail.com

Received April 29, 2015; Accepted June 12, 2015; Published June 18, 2015

Citation: Hussain S, Rather H, Qayoom A (2015) Efficacy, Tolerability and Adverse Events of Single-Shot Intra-Articular Hyaluronic Acid Injection in Knee Osteoarthritis. J Trauma Treat 4: 256. doi:10.4172/2167-1222.1000256

Copyright: (c) 2015 Hussain S, et al. This is an open-access article distributed under the terms of the Creative Commons Attribution License, which permits unrestricted use, distribution, and reproduction in any medium, provided the original author and source are credited. 
The exact mechanism by which intra-articular HA or hylans act is currently unknown. Studies have shown the direct analgesic effect of hyaluronic acid in a rat model [8]. The authors proposed that hyaluronic acid modulates pain by directly inhibiting nociceptors or indirectly binding substance $\mathrm{P}$, which is involved in pain signals. HA blocks the loss of prostaglandins from the cartilage matrix into the synovial fluid, maintaining the normal cartilage structure [3]. Exogenous HA may facilitate chondrocyte proliferation and de novo synovial synthesis of HAs $[9,10]$. Relief of knee pain from OA with HA in clinical studies may be due to the effects of HA on nerve impulses and nerve sensitivity [11] and suppression of inflammatory responses to interleukin-1 $[12,13]$. HA cushions and adds flexibility to cartilage, thus making it resilient to compression.

Presently, HA is indicated for patients with knee OA who fail to respond to conservative measures like physical therapy and pharmacological agents including NSAIDS and interleukin-1 inhibitors etc [14]. This is corroborated with clinical trials comparing HA and placebo, where in pain relief was significant with HA [15]. Amelioration of pain is comparable with NSAIDS [16] and even superior to intraarticular corticosteroid [17], lasts longer but takes more time to take effect [16]. This latter finding may be especially advantageous in patients in whom typical pain killers are contraindicated or in those who have experienced either a lack of efficacy or other adverse events (patients with peptic ulcer or history of gastrointestinal bleed).

Given the chronic nature of osteoarthritis, the potential utility of HA is currently limited by the paucity of data concerning not only the effectiveness in the short term [18] but the effectiveness of longterm multiple courses of intraarticular HA therapy [19]. The literature on single shot HA injections is even sparser. The purpose of this study was to evaluate both clinical and functional outcomes, patient satisfaction, adverse events and need for concomitant analgesia for knee osteoarthritis after single shot HA injection.

\section{Materials and methods}

\section{Subjects}

This single-center prospective clinical trial was approved by the Institute ethical committee. Forty-eight (48) patients were enrolled between Feb 2008 and Dec 2010 (Table 1). All patients had symptomatic knee pain at the tibiofemoral joint and radiographic evidence of osteoarthritis (loss of cartilage thickness, osteophyte

\begin{tabular}{|l|c|}
\hline & Number \& Percentage \\
\hline Total number of patients & 48 \\
\hline Sex & $27(56.2 \%)$ \\
\hline Female, $\mathrm{n}(\%)$ & $21(43.7 \%)$ \\
\hline Males, $\mathrm{n}(\%)$ & \\
\hline Age & $65( \pm 5)$ \\
\hline Mean (SD) & 55 to 70 \\
\hline Range & $29.1(4.8)$ \\
\hline Body Mass Index $\left(\mathbf{k g} / \mathbf{m}^{\mathbf{2}}\right)$ & \\
\hline Mean (SD) & \\
\hline Knee OA & \\
\hline Grading as per Kellgren-Lawrence Classification & $30(62.5 \%)$ \\
\hline \multicolumn{1}{|c|}{ Grade I } & $18(37.5 \%)$ \\
\hline Grade II & 0 \\
\hline Grade III & \\
\hline Grade IV & \\
\hline
\end{tabular}

Table 1: Patient demographics. formation, subchondral sclerosis, or cysts) [20]. All patients had received conservative treatment for knee pain in the form of NSAIDS, interleukin-1 inhibitors and physical therapy (ultrasound therapy, short-wave diathermy) with unsatisfactory results.

The patients with unilateral osteoarthritis knee were observed after a single shot intraarticular HA injection with synvisc-One. Although some patients ( $\mathrm{n}=22,45.8 \%)$ had osteoarthritis in the contralateral knee, only the painful and functionally limiting knee was studied. At entry, the affected knee had radiographic evidence of grade 2-3 osteoarthritis [21]. Patients with grade 4 arthritis and those who had received previous intraarticular HA or glucocorticoid injection were excluded. Patients with inflammatory joint disease, crystal arthropathy, suspicion of infection in or around the affected knee, history of arthroplasty and coagulopathy were also excluded.

\section{Assessment}

Patient details and demographic data were documented (age, sex, body mass index, comorbidities, and concomitant medications). The WOMAC [22] and VAS pain scores were noted. The WOMAC scores range from 0 (best score) to 96 (worst score) based on the three subdomains of pain, stiffness, and difficulty in performing daily activities. The visual analog scale (VAS) ranged from 0 to $100 \mathrm{~mm}$, with lower numbers representing less pain and higher numbers representing more pain. The VAS and WOMAC was used as a self-administered questionnaire in accordance with the developers' instructions [22]. Patient satisfaction was determined as per Linkert scale [23] (Figure 1).

Objective analysis included measurement of knee flexion-extension, circumference at the level of suprapatellar pouch and change in tibiofemoral joint space width.

\section{Intervention}

Synvisc-One is supplied as $6 \mathrm{ml}$ syringe containing hylan G-F 20 and was purchased by patients from market. Synvisc-One was administered as a single shot injection as per manufacturer's instructions. Under aseptic precautions, the injections were performed via supero-lateral approach with a 22-gauge needle in minor OT. Patients were instructed to refrain from strenuous activity for a day following the intra-articular injections. Synovial fluid analysis was done in 15 Patients.

Patients were instructed to wait a full month following the injection of synvisc-One in order to let the product sufficient time to take effect. Patients were then followed-up at 1, 3 and 6 months with final follow up at 1 year.

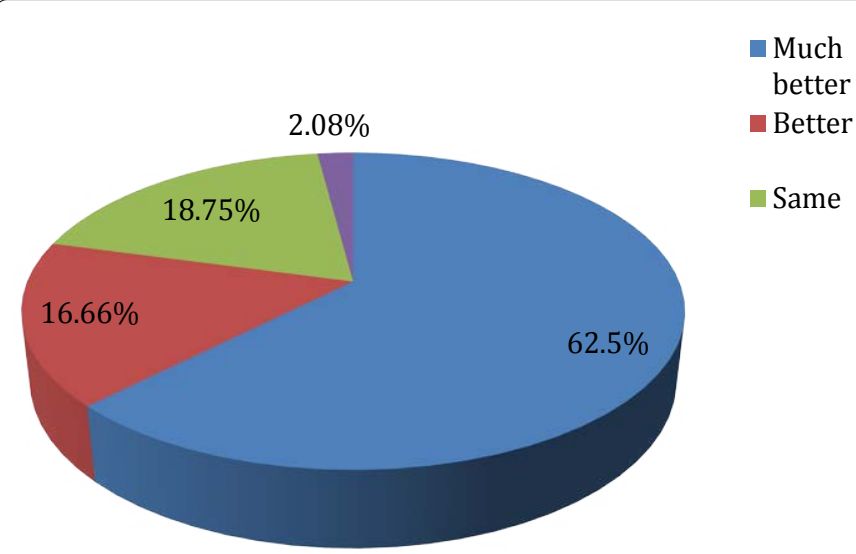

Figure 1: Patient satisfaction at 6 months after hyaluronic acid injection. 


\section{Results}

\section{Subject Characteristics}

Forty-eight (48) patients were recruited for the study over a period of 2 years. Study population baseline demographics are given in Table 1. No patient was lost to follow up. Majority of the patients were females (56.2\%). 30 patients (62.5\%) described one or more comorbid condition at baseline including controlled hypertension, hypothyroidism, diabetes mellitus and acid dyspepsia etc. $80 \%$ patients had used NSAIDS, 50\% had used diacerin while $35 \%$ had received physical modality at some point of time.

\section{Outcome}

A. Evaluation of visual analog pain scale (VAS).

1. VAS at rest: Visual analog pain scale (VAS) revealed an average score of 70 pre-injection at rest. From this base line, the value dropped to 45

2. VAS after walking: Walking $30 \mathrm{~m}$ revealed an average pain score of 82 pre-injection that dropped to 50 .

B. The WOMAC scores improved with a median decrease from 60 to 44 points.

At 6 months, $79.1 \%$ patients (38) improved with viscosupplementation while $9(18.75 \%)$ remained the same. One patient (2\%) went onto develop septic arthritis managed with intravenous antibiotics and arthrotomy and constituted the poor result in our study. The scores however returned to baseline at the end of 1 year.

C. Substantial improvement was noted in knee flexion-extension and knee swelling $(\mathrm{p}<0.05)$ but no change was observed in joint space width.

D. Secondary outcome measures included patient satisfaction as per Linkert scale [23] and frequency of local adverse events (effusions or flares) in injected knees (Figure 2). (79.1\%) patient satisfaction with the HA was significant. The patient satisfaction remained so at 1 year also with patients quoting good symptomatic relief with HA injection even though VAS and WOMAC scores indicating otherwise. There were no systemic adverse events reported. Local adverse events including pain and swelling at injection site were observed in injections (Figures 2 and 3). The need for analgesia was reduced and activities of daily living improved. Patients with lower body mass index had better results.

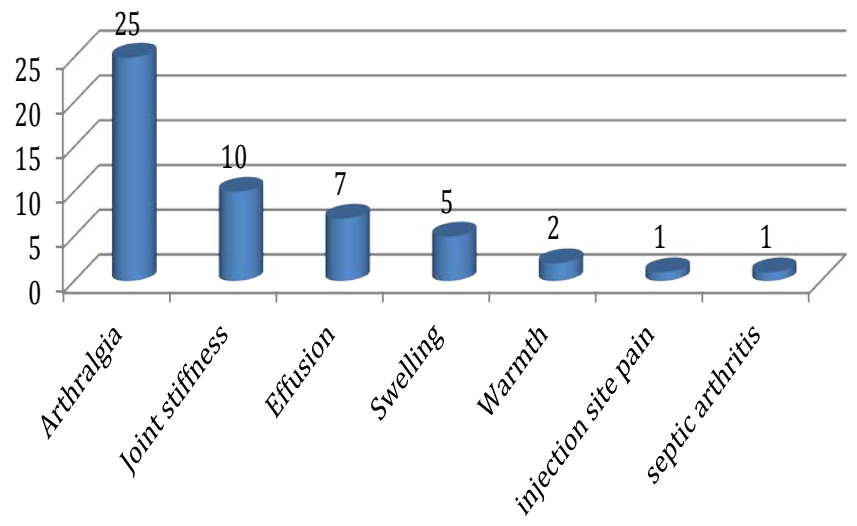

Figure 2: Adverse events of single-shot hyaluronic acid injection (Note that some patients had more than 1 adverse event while some had none).

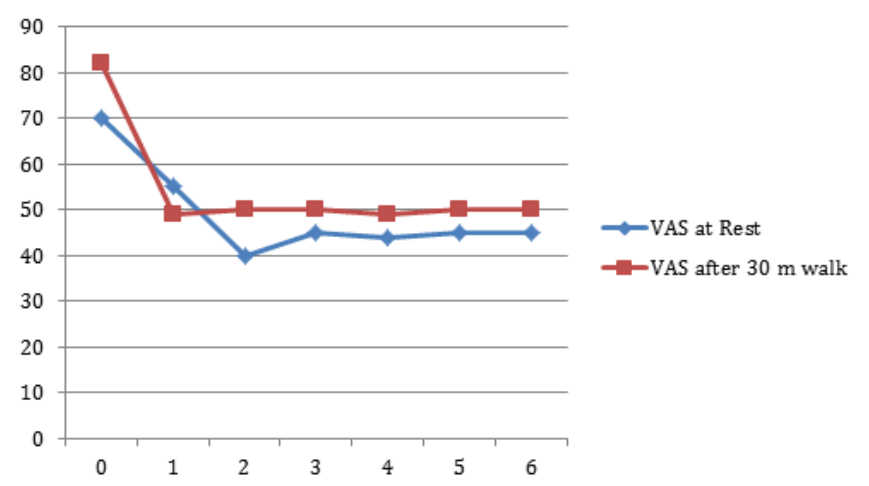

Months post treatment

Figure 3: Change in VAS score.

\section{Discussion}

Osteoarthritis is the most common form of joint disorder in the elderly and a major source of disability [1]. The prevalence continues to increase as the older adult and obese populations grow $[14,15]$. More than $50 \%$ of patients older than 65 years have radiographic changes in the knee that indicates arthritis [15]. It is characterized by activity related pain and troublesome stiffness. Radiological analysis often reveals progressive joint space narrowing, osteophyte formation, bony enlargement and in advanced cases, misalignment of anatomical/ mechanical axis. The etiology is multifactorial with genetic and environmental factors playing important roles. Advancing age and obesity are important predisposing risk factors for primary osteoarthritis $[24,25]$. Weight bearing joints like knee, hip, and ankle are commonly involved. In the knee, pain is usually the presenting symptom followed by progressive loss of motion, joint tenderness, effusion and stiffness, and in the later stages, joint deformity. OA knees is often bilateral though symptoms appear in one joint initially.

Traditional nonoperative treatment of osteoarthritis includes activity modification, weight loss, rehabilitative exercises, nonsteroidal anti-inflammatory medications (NSAIDs) and intra-articular injections. Surgical treatment, in the form of osteotomy and arthroplasty of the hip and knee is effective, but not indicated for early stages of the disease in all patients. The enormous costs involved and relevance of such procedures in third world setting as ours is debatable.

The first line treatment usually is begun with acetaminophen and NSAIDS, but the treatment is associated with significant morbidity to the gastrointestinal system and renal function [25]. Interleukin-1 inhibitors are widely used but often associated with troublesome diarrhea [26]. Intraarticular corticosteroid injections for the treatment of osteoarthritic knees are well documented. The symptom relief is prompt but short-lived. Some studies suggest that intraarticular corticosteroid injections result in severe deleterious effects, both mechanical and physiological, on the joint and articular cartilage [27]. Corticosteroid injections can cause skin depigmentation/ atrophy, ligament and tendon rupture and dreaded complication of sepsis. At higher doses, which occur when treating multiple joints, the corticosteroids can inhibit the hypothalamic-pituitary-adrenal axis [28].

Visco-supplementation with HA has gained popularity as a modality for the treatment of osteoarthritis of the knee. A Cochrane analysis of visco-supplementation for the treatment of knee OA found HA derivatives as a class to be effective $[29,30]$. These injections 
provide a combination of viscoelastic properties with associated antiinflammatory, anabolic, and chondro-protective effects [29] protecting the joint from compressive and shear forces. The content of synovial fluid, in the presence of osteoarthritis, has a decreased concentration and molecular weight of hyaluronic acid. The rationale for the intraarticular injection of hyaluronic acid is to restore the viscoelasticity of synovial fluid by providing exogenous HA thus helping to augment the flow of synovial fluid, normalize the synthesis and inhibit the degradation of endogenous hyaluronic acid, and relieve joint pain $[15,30]$. Hyaluronic acid also has several anti-inflammatory effects by inhibiting phagocytosis and adherence of leukocytes. It reduces the levels of inflammatory mediators such as prostaglandin and cyclic adenosine monophosphate. Hyaluronic acid also reduces the release of arachidonic acid from synovial fibroblasts [28]. The properties of biocompatibility and a prolonged half-life within the synovium are added advantages [29].

The literature supports the use and efficacy of HA even though AAOS hasn't recommended for or against it. Many randomized controlled studies have demonstrated a longer-term effect in pain control with HA injections compared with corticosteroids [31] and placebo [32,33]. Hyaluronic acid has approximately a $1 \%$ incidence of side effects per injection. The most common side effects are local reactions of the knee such as swelling, pain, and increased warmth. This type of reaction typically lasts for 1 to 2 days [7]. With proper patient selection and injection technique, these injections are a viable treatment option for patients with early symptomatic knee OA. The group receiving the hyaluronic acid also had a significantly reduced need for treatment at 1 year [33].

The present study suggests beneficial effects of hyaluronic acid in patients with Kellgren-Lawrence grade 2 osteoarthritis more than grade 3 osteoarthritis. The need for concomitant analgesia like NSAIDS, opiods etc and modalities like ultrasound therapy, short wave diathermy etc is reduced. There is increasing interest in the use of HAs for OA at sites other than the knee including shoulder and hip OA $[34,35]$.

\section{Conclusion}

The results of this study show that a single intraarticular injection of hyaluronic acid has a significant effect on functional outcome and reduction of osteoarthritic knee pain. The duration of symptom relief for upto 6 months suggests a possible disease modifying effect of HA in knee osteoarthritis. Patient selection is important to achieve satisfactory results. The response to injection in early OA (Grade 2 ) is significantly better than in advanced arthritis. Advancing age and obesity seems to be unfavorable variables for symptomatic improvement. The procedure is associated with few local adverse events and decreases the need for concomitant analgesia/ therapeutic modalities. Activity related pain seems to respond better to HA injection which is similar to previous studies [36]. The need for long-term study cannot be over emphasized. This is particularly so since no change was observed in joint space width over the study period of one year.

Even though the literature is replete with data on multiple intraarticular hyaluronic acid injections in knee osteoarthritis, knowledge on single-shot injections is sparse. When compared with these studies, single-shot hyaluronic acid injections seem equally effective. The lesser hospital visits and procedures is another advantage. The financial implications of multiple hospital visits is a concern in our setup, a single visit is obviously beneficial for the patient. A single infection also subjects the patient to less trauma compared to multiple injections.

\section{References}

1. Dieppe P (1998) Osteoarthritis. Acta Orthop Scand Suppl 281: 2-5.

2. Balazs EA, Denlinger JL (1993) Viscosupplementation: a new concept in the treatment of osteoarthritis. J Rheumatol Suppl 39: 3-9.

3. No authors listed (2000) Recommendations for the medical management of osteoarthritis of the hip and knee: 2000 update. American College of Rheumatology Subcommittee on Osteoarthritis Guidelines. Arthritis Rheum 43 1905-1915.

4. Laurent TC, Fraser JR (1992) Hyaluronan. FASEB J 6: 2397-2404.

5. Balazs E (1982) The physical properties of synovial fluid and the specific role of hyaluronic acid. In Disorders of the Knee. In: Helfet AJ (ed). Philadelphia: J B Lippincott 61-74

6. Belcher C, Yaqub R, Fawthrop F, Bayliss M, Doherty M (1997) Synovial fluid chondroitin and keratan sulphate epitopes, glycosaminoglycans, and hyaluronan in arthritic and normal knees. Ann Rheum Dis 56: 299-307.

7. Rydell N, Balazs EA (1971) Effect of intra-articular injection of hyaluronic acid on the clinical symptoms of osteoarthritis and on granulation tissue formation. Clin Orthop Relat Res 80: 25-32.

8. Ghosh $\mathrm{P}$ (1994) The role of hyaluronic acid (hyaluronan) in health and disease: interactions with cells, cartilage and components of synovial fluid. Clin Exp Rheumatol 12: 75-82.

9. Smith MM, Ghosh P (1987) The synthesis of hyaluronic acid by human synovial fibroblasts is influenced by the nature of the hyaluronate in the extracellular environment. Rheumatol Int 7: 113-122.

10. Bagga $H$, Burkhardt D, Sambrook $P$, March $L$ (2006) Longterm effects of intraarticular hyaluronan on synovial fluid in osteoarthritis of the knee. J Rheumatol 33: 946-950.

11. Begg C, Cho M, Eastwood S, Horton R, Moher D, et al (1996) Improving the quality of reporting clinical trials: The CONSORT statement. JAMA 276: 637639.

12. Yasui T, Akatsuka M, Tobetto K, Hayaishi M, Ando T (1992) The effect of hyaluronan on interleukin-1 alpha-induced prostaglandin E2 production in human osteoarthritic synovial cells. Agents Actions 37: 155-156.

13. Monfort J, Nacher M, Montell E, Vila J, Verges J, et al. (2005) Chondroitin sulfate and hyaluronic acid (500-730 kda) inhibit stromelysin-1 synthesis in human osteoarthritic chondrocytes. Drugs Exp Clin Res 31: 71-76.

14. Kim RH, Springer BD, Douglas DA (2011) Knee reconstruction and replacement In: Flynn F, ed. Orthopaedic Knowledge Update. Rosemont, IL: American Academy of Orthopaedic Surgeons 469-475.

15. Arden N, Nevitt MC (2006) Osteoarthritis: epidemiology. Best Pract Res Clin Rheumatol 20: 3-25.

16. Adams ME, Atkinson MH, Lussier AJ, Schulz JI, Siminovitch KA, et al. (1995) The role of visco-supplementation with hylan GF-20 (Synvisc) in the treatment of osteoarthritis of the knee: A Canadian multi-center trial comparing Hylan GF20 alone, hylan GF-20 with non-steroidal anti-inflammatory drugs (NSAIDS) and NSAIDS alone. Osteoarthritis Cartilage 3: 213-226.

17. Mamlin LA, Melfi CA, Parchman ML, Gutierrez B, Allen DI, et al. (1998) Management of osteoarthritis of the knee by primary care physicians. Arch Fam Med 7: 563-567.

18. Lo GH, LaValley M, McAlindon T, Felson DT (2003) Intra-articular hyaluronic acid in treatment of knee osteoarthritis: a meta-analysis. JAMA 290: 3115-3121.

19. Lussier A, Cividino AA, McFarlane CA, Olszynski WP, Potashner WJ, et al. (1996) Viscosupplementation with hylan for the treatment of osteoarthritis: findings from clinical practice in Canada. J Rheumatol 23: 1579-1585.

20. Altman R, Asch E, Bloch D, Bole G, Borenstein D, et al. (1986) Development of criteria for the classification and reporting of osteoarthritis. Classification of osteoarthritis of the knee. Diagnostic and Therapeutic Criteria Committee of the American Rheumatism Association. Arthritis Rheum 29: 1039-1049.

21. Kellgren JH, Jeffrey MR, Ball J (1963) The epidemiology of chronic rheumatism. Atlas of standard radiographs of arthritis. Oxford: Blackwell Scientific Publications.

22. Bellamy N (2000) WOMAC osteoarthritis index users guide. University of Queensland, Queensland, Australia 1-43. 
Citation: Hussain S, Rather H, Qayoom A (2015) Efficacy, Tolerability and Adverse Events of Single-Shot Intra-Articular Hyaluronic Acid Injection in Knee Osteoarthritis. J Trauma Treat 4: 256. doi:10.4172/2167-1222.1000256

Page 5 of 5

23. Linkert R (1932). "A Technique for the Measurement of Attitudes". Archives of Psychology 140: 1-55.

24. Deyle GD, Allison SC, Matekel RL, Ryder MG, Stang JM, et al. (2005) Physical therapy treatment effectiveness for osteoarthritis of the knee: a randomized comparison of supervised clinical exercise and manual therapy procedures versus a home exercise program. Phys Ther 85: 1301-1317.

25. Fries JF (1998) The epidemiology of NSAID gastropathy: the ARAMIS experience. J Clin Rheumatol 4: s11-16.

26. Bartels EM, Bliddal H, Schøndorff PK, Altman RD, Zhang W, et al. (2010) Symptomatic efficacy and safety of diacerein in the treatment of osteoarthritis: a meta-analysis of randomized placebo-controlled trials. Osteoarthritis and Cartilage 18: 289-296.

27. Hauser RA (2009) The Deterioration of Articular Cartilage in Osteoarthritis by Corticosteroid Injections. Journal of Prolotherapy 1.

28. Snibbe JC, Gambardella RA (2005) Use of injections for osteoarthritis in joints and sports activity. Clin Sports Med 24: 83-91.

29. Watterson JR, Esdaile JM (2000) Viscosupplementation: therapeutic mechanisms and clinical potential in osteoarthritis of the knee. J Am Acad Orthop Surg 8: 277-284.

30. Lee S, Park D, Chmell SJ (2004) Viscosupplementation with hylan G-F 20
(Synvisc): pain and mobility observations from 74 consecutive patients. J Knee Surg 17: 73-77.

31. Bellamy N, Campbell J, Robinson V, Gee T, Bourne R, et al. (2006) Viscosupplementation for the treatment of osteoarthritis of the knee. Cochrane Database Syst Rev 19: CD005321.

32. Dixon AS, Jacoby RK, Berry H, Hamilton EB (1988) Clinical trial of intra-articular injection of sodium hyaluronate in patients with osteoarthritis of the knee. Curr Med Res Opin 11: 205-213.

33. Dougados M, Nguyen M, Listrat V, Amor B (1993) High molecular weight sodium hyaluronate (hyalectin) in osteoarthritis of the knee: a 1 year placebocontrolled trial. Osteoarthritis Cartilage 1: 97-103.

34. Brander VA, Gomberawalla A, Chambers M, Bowen M, Nuber G (2010) Efficacy and safety of hylan G-F 20 for symptomatic glenohumeral osteoarthritis: a prospective, pilot study. PM R 2: 259-267.

35. Migliore A, Tormenta S, Massafra U, Carloni E, Padalino C, et al. (2005) Repeated ultrasound-guided intra-articular injections of $40 \mathrm{mg}$ of Hyalgan may be useful in symptomatic relief of hip osteoarthritis. Osteoarthritis Cartilage. 13: $1126-1127$.

36. Petrella RJ, DiSilvestro MD, Hildebrand C (2002) Effects of hyaluronate sodium on pain and physical functioning in osteoarthritis of the knee: a randomized double-blind, placebo-controlled clinical trial. Arch Intern Med 162: 292-298. 